\title{
Effect of multiple courses of antenatal corticosteroids on pituitary-adrenal function in preterm infants
}

\author{
P C Ng, G W K Wong, C W K Lam, C H Lee, T F Fok, M Y Wong, K C Ma
}

\begin{abstract}
Aim-To evaluate the pituitary-adrenal function of preterm infants whose mothers received multiple courses (8 or more doses) of antenatal dexamethasone.

Methods-The pituitary-adrenal function of 14 preterm infants whose mothers received eight or more doses of antenatal dexamethasone were assessed using the human corticotrophin releasing hormone (hCRH) stimulation test when 7 days $(n=$ 14) and 14 days old $(n=12)$. During each test, blood samples were taken at 0 (baseline), 15, 30 and 60 minutes after an intravenous bolus dose of hCRH $(1 \mu \mathrm{g} / \mathrm{kg})$. The corresponding hormone concentrations were compared between days 7 and 14 , and with various associated factors.

Results-The baseline (0 min) plasma adrenocorticotrophic hormone concentration was significantly higher at day 14 than at day $7(p=0.036)$. None of the corresponding poststimulation $(15,30$, and 60 min) hormone concentrations was significantly different between the two time epochs. When the association between the hormone concentrations and the number of antenatal dexamethasone doses received by the mothers was assessed, a significant negative correlation was observed in serum cortisol concentrations at 15 and 30 min on day $14(r=-0.59, p=0.04$ and $r$ $=-0.60, \mathrm{p}=0.039$, respectively).
\end{abstract}

Conclusions-The absence of a significant difference in poststimulation hormone concentrations between days 7 and 14 in this cohort of infants, and the similarity of their hormone responses with those of older children and adults, suggests that no severe pituitary-adrenal suppression had occurred. None the less there was evidence of mild adrenal suppression in some of the treated infants. Vigilance in monitoring blood pressure, electrolytes and signs of adrenal suppression in infants whose mothers receive multiple courses ( 8 or more doses) of antenatal dexamethasone is required, as some of them might have diminished adrenal reserve.

(Arch Dis Child Fetal Neonatal Ed 1999;80:F213-F216)

Keywords: antenatal dexamethasone; hCRH; pituitaryadrenal; preterm

Antenatal corticosteroids have been widely used for the prevention of respiratory distress syndrome $(\mathrm{RDS})^{12}$ and its associated complications $^{3-5}$ in preterm infants. This modality of treatment has become an established part of routine obstetric management in many institutions, but as the effectiveness of the treatment wanes after 7 days, ${ }^{6}$ repeated courses of corticosteroids are required to sustain the beneficial effect if the risk of imminent preterm delivery persists or recurs. We have recently studied the influence of antenatal and postnatal dexamethasone on the hypothalamicpituitary-adrenal (HPA) axis in preterm, very low birthweight (VLBW) infants ${ }^{78}$ and our results suggest that infants whose mothers received less than eight doses (4 courses) of antenatal dexamethasone, have no long lasting suppressive effects on the pituitary-adrenal function at day 7 or later. ${ }^{8}$ We have also shown that both the pituitary and adrenal glands had substantially recovered 28 days after discontinuation of a three week course of postnatal dexamethasone during which the dose was gradually reduced. ${ }^{7}$ As we were unsure whether eight or more doses of antenatal dexamethasone would suppress the HPA axis, a prospective study was performed to investigate the effect of multiple courses ( 8 or more doses) of antenatal dexamethasone on the pituitaryadrenal function using the human corticotrophin releasing hormone $(\mathrm{hCRH})$ stimulation test.

\section{Methods}

Fourteen preterm infants were prospectively recruited between August 1994 and December 1997. Inclusion criteria were: (i) infants whose mother received eight or more doses of antenatal dexamethasone during pregnancy; (ii) time interval between the last dose of antenatal steroid and delivery less than 28 days; (iii) presence of an intravascular line at day 7 (a second hCRH test would also be performed if the intravascular line was still in situ on day 14); and (iv) no postnatal inhaled or systemic

Table 1 Clinical characteristics of study population $(n=14)$

\begin{tabular}{ll}
\hline Gestational age (weeks) $\dagger$ & $32.5(2.3)$ \\
Birth weight $(\mathrm{g}) \dagger$ & $1539(438)$ \\
Apgar scores $1 \mathrm{~min}<7(\mathrm{n}), 5 \mathrm{~min}<7(\mathrm{n})$ & 3,0 \\
$\begin{array}{l}\text { Number of antenatal dexamethasone doses } \\
\quad \text { received }\end{array}$ & $10(8-18)$ \\
Time between the last dose of antenatal steroid & \\
$\quad$ and delivery (h) & $102(18-432)$ \\
Mode of delivery: vaginal: caesarean section & $1: 13$ \\
Male:female & $7: 7$ \\
Singleton:twins:triplets & $8: 0: 6$ \\
Inborn:outborn & $14: 0$ \\
Infants requiring assisted ventilation at day 7 & \\
$\quad(\mathrm{n})$, at day $14(\mathrm{n})$ & 5,3 \\
\hline
\end{tabular}

† values are mean $(\mathrm{SD}) ;{ }^{\star}$ values are median (range) 
Table 2 Plasma ACTH and serum cortisol concentrations before and after hCRH stimulation at days 7 and 14 in preterm infants whose mothers received eight or more doses of antenatal dexamethasone

\begin{tabular}{|c|c|c|c|c|c|c|c|c|}
\hline & \multicolumn{4}{|c|}{$A C T H(p m o l / l)$} & \multicolumn{4}{|c|}{ Cortisol (nmol/l) } \\
\hline & $0 \mathrm{~min}$ & 15 mins & 30 mins & 60 mins & $0 \mathrm{~min}$ & 15 mins & 30 mins & 60 mins \\
\hline Day $7(n=14)$ & $5.1(0.7)$ & $11.5(2.3)$ & $8.4(1.4)$ & $6.3(0.9)$ & $283(47)$ & $450(44)$ & $552(60)$ & $347(64)$ \\
\hline Day $14(\mathrm{n}=12)$ & $7.9(1.1)$ & $12.0(2.4)$ & $10.2(1.6)$ & $8.3(1.3)$ & $371(33)$ & $453(46)$ & $552(56)$ & $374(50)$ \\
\hline \multicolumn{9}{|c|}{ Differences between days } \\
\hline 14 and $7(n=12)$ & $2.4(1.3)$ & $2.1(2.3)$ & $2.8(1.3)$ & $2.6(1.1)$ & $62(61)$ & $-12(78)$ & $-20(86)$ & $25(96)$ \\
\hline
\end{tabular}

Values are mean (SEM)

corticosteroids treatment before the hCRH tests. Infants were excluded if they had concurrent hypoglycaemia, systemic infection, necrotising enterocolitis, or major surgery in the preceding week.

The decision to administer antenatal dexamethasone rested entirely with the attending obstetrician. Management guidelines for starting antenatal dexamethasone treatment in women between $24-34$ weeks of gestation were: (i) threatened preterm labour; (ii) antepartum haemorrhage; (iii) preterm rupture of membranes; and (iv) any condition requiring elective premature delivery. Repeated courses were administered weekly if the risk of imminent preterm delivery persisted or recurred after initial treatment. Treatment was to be stopped when the fetus reached 34 weeks of gestation as the risk of developing severe RDS was minimal after this critical period. Each course consisted of two doses of dexamethasone (dexamethasone sodium phosphate; Weimer Pharma, Gmbh, Rastatt, Germany) 10 mg given intramuscularly 12 hours apart.

We performed the hCRH stimulation test following a standard schedule on days 7 and 14. ${ }^{89}$ The hCRH test was performed between 0800-1000 hours, as described before. ${ }^{9}$ The plasma corticotrophin (ACTH) and serum cortisol concentration were measured by double antibody radioimmunoassay and solid phase radioimmunoassay, respectively. ${ }^{9}$

Ethical approval for the study was obtained from the Research Ethics Committee of the Chinese University of Hong Kong. Informed parental consent was obtained for each case before the start of any test.

Student's $t$ test was used to compare the corresponding hormone concentrations between days 7 and 14 of postnatal age. Linear regression analysis was used to assess the correlation between the hormone concentrations and various associated factors.

\section{Results}

Fourteen infants whose mothers received eight or more doses (mean 11.6; median 10; range 8-18 doses) of antenatal dexamethasone were enrolled. The clinical characteristics of the study population are summarised in table 1 . Twenty six hCRH stimulation tests were performed in this cohort of infants at days 7 and 14 of postnatal age. Two infants missed the second hCRH test on day 14 as they no longer had an intravascular line. Table 2 and figs 1 and 2 show the mean plasma ACTH and serum cortisol concentrations in response to hCRH stimulation at days 7 and 14 .

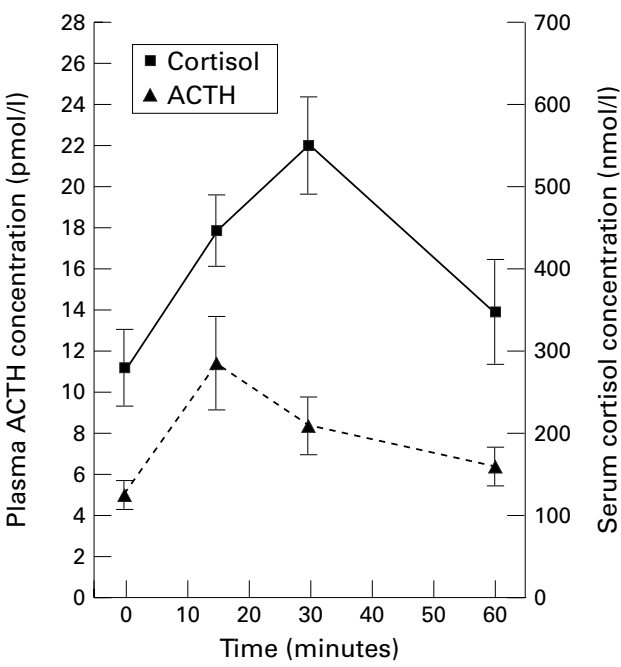

Figure 1 Effect of exogenous hCRH stimulation on plasma ACTH and serum cortisol concentrations at day 7 of postnatal age in preterm infants $(n=14)$ whose mother received eight or more doses of antenatal dexamethasone. Values are mean (SEM).

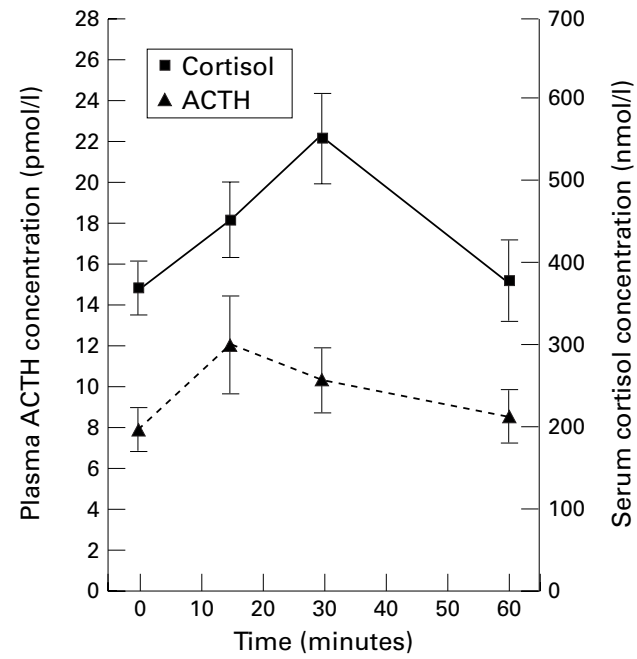

Figure 2 Effect of exogenous hCRH stimulation on plasma ACTH and serum cortisol concentrations at day 14 of postnatal age in preterm infants $(n=12)$ whose mother received eight or more doses of antenatal dexamethasone. Values are mean (SEM).

The baseline ( 0 min) plasma ACTH concentration was significantly higher at day 14 than at day $7(p=0.036)$. None of the poststimulation $(15,30$, and $60 \mathrm{~min})$ plasma ACTH and serum cortisol concentrations at day 14 was significantly different when compared with their corresponding concentrations at day $7(\mathrm{p}$ $>0.05$ and $p>0.15$, respectively).

A negative trend was observed when the association between the hormone concentra- 
tions (at each individual sampling time and collectively as area under the curve) and the number of antenatal dexamethasone doses received by the mothers was assessed at days 7 and 14. This correlation was significant for serum cortisol concentrations at 15 and $30 \mathrm{~min}$ on day $14(\mathrm{r}=-0.59, \mathrm{p}=0.04$ and $\mathrm{r}=-0.60, \mathrm{p}$ $=0.039$, respectively). A negative trend was also observed when the correlation between the hormone concentrations and the time intervals between the last dose of antenatal dexamethasone and delivery was assessed at day 7 . However, none of the correlation coefficients reached significance and this negative trend was no longer observed by day 14 of postnatal age.

\section{Discussion}

One of the most feared adverse effects of antenatal dexamethasone is HPA axis suppression in newborn infants. As this treatment is of confirmed value for the prevention of $\mathrm{RDS},{ }^{12}$ perinatal clinicians are likely to be more liberal in prescribing multiple courses of antenatal corticosteroids to mothers who are at high risk of imminent preterm delivery. However, little is known about HPA axis suppression in preterm infants whose mothers are given eight or more doses. Hence this study was undertaken to investigate in detail the effect on the pituitaryadrenal function in such patients.

A major problem in assessing the adequacy of the pituitary-adrenal response in this cohort of moderately premature infants is the lack of a reference standard against which their hormone responses may be judged. ${ }^{10}$ It would not be ethical to include a control group by subjecting infants of gestational age 32 weeks or above, who had never received antenatal corticosteroids, to the hCRH stimulation test. Most such patients would not require intravascular line monitoring and their chance of requiring postnatal corticosteroid treatment should be minimal. In the absence of an ideal control group we opted to compare these results with those of our previous work which involved 17 preterm VLBW infants who did not receive antenatal corticosteroids and who had their pituitary-adrenal function assessed by the hCRH test at days 7 and 14 of life. ${ }^{8}$ Only the 60 min serum cortisol concentration at day 7 was significantly lower in the antenatal corticosteroid treatment group than in the non-treatment group when corresponding hormone concentrations were compared (mean (SEM) serum cortisol concentrations were 347 (64) and 632 (83) nmol/1, respectively; mean (SEM) differences were 285 (108) nmol/1; p < 0.02). Obviously, such comparison should be interpreted with caution as the demographic characteristics between the two groups of infants were different. None the less it does provide an insight into the HPA function of preterm infants and, more importantly, we did not find any severe pituitaryadrenal suppression in the treated neonates. Furthermore, the configuration of the response curves and the magnitude of the responses (figs 1 and 2) were similar to those observed in older children and adults. ${ }^{11} 12$
There were also no significant differences in poststimulation plasma ACTH and serum cortisol concentrations between days 7 and 14 in the studied population (table 2), providing further evidence that the infants' HPA axis remained responsive despite multiple courses of antenatal corticosteroids. The higher baseline plasma ACTH concentration at day 14 probably represented the postnatal adaptation of the endocrine axis after birth. ${ }^{8}{ }^{13}$

Despite the above findings there was evidence of mild adrenal suppression in infants whose mother received multiple courses of antenatal dexamethasone. A significant negative correlation was observed between the poststimulation serum cortisol concentrations of the infants and the cumulative antenatal dexamethasone doses received by the mothers. Furthermore, three of 26 hCRH tests had peak serum cortisol concentrations < $330 \mathrm{nmol} / 1$, which suggested slight blunting of the adrenal responses. Nevertheless, it was reassuring to note that none of the studied infants developed clinical signs or electrolyte disturbances suggestive of adrenal insufficiency, or required steroid replacement.

In summary, multiple courses (mean 11.6 doses) of antenatal dexamethasone do not cause severe pituitary-adrenal suppression in neonates at 7 days of age or older. However, a mild degree of adrenal suppression may occur in a small proportion of treated infants. Although the severity of suppression increases with the cumulative dexamethasone dose received by the mothers, the effect does not translate into clinically significant adrenal insufficiency or crisis. We can confidently conclude from our series of studies ${ }^{7-9}$ that a standard course of antenatal dexamethasone has no long lasting suppressive effects on the pituitaryadrenal function in newborn infants. Even multiple courses (mean 11.6 doses) of antenatal corticosteroids have relatively little clinical or biochemical influence on the endocrine axis. However, we urge for vigilance in monitoring blood pressure, electrolytes, and signs of adrenal suppression in all neonates whose mothers have received eight or more doses of antenatal dexamethasone, as some of these infants might have diminished adrenal reserve.

1 Crowley PA. Antenatal corticosteroid therapy: a metaanalysis of the randomized trials, 1972 to 1994 . Am f Obstet Gynecol 1995;173:322-35.

2 Sinclair JC. Meta-analysis of randomized controlled trials of antenatal corticosteroid for the prevention of respiratory distress syndrome: discussion. Am f Obstet Gynecol 1995;173:335-44

3 Van Marter LJ, Leviton A, Kuban KCK, Pagano M, Allred EN. Maternal glucocorticoid therapy and reduced risk of bronchopulmonary dysplasia. Pediatrics 1990; 86:331-6.

4 Leviton A, Kuban KCK, Pagano M, Allred EN, Van Marter LJ. Antenatal corticosteroids appear to reduce the risk of LJ. Antenatal corticosteroids appear to reduce the risk of
postnatal germinal matrix hemorrhage in intubated low postnatal germinal matrix hemorrhage in intubat
birth weight newborns. Pediatrics 1993;91:1083-8.

5 Beuer JC, Morrison J, Poole W. Decreased incidence of necrotizing enterocolitis after prenatal glucocorticoid therapy. Pediatrics 1984;73:682-8.

6 Howie RN, Liggins GC. The New Zealand study of antepartum glucocorticoid treatment. In: Farrell PM, ed. Lung Development: biological and clinical prospective. Vol 2. New York: Academic Press, 1982: 255-65.

7 Ng PC, Wong GWK, Lam CWK, et al. Pituitary-adrenal suppression and recovery in preterm very low birth weight infants after dexamethasone treatment for bronchopulmonary dysplasia. f Clin Endocrinol Metab 1997;82:2429-32.

$8 \mathrm{Ng}$ PC, Wong GWK, Lam CWK, et al. Pituitary-adrenal response in preterm very low birth weight infants after 
treatment with antenatal corticosteroids. $\mathcal{F}$ Clin Endocrinol

Metab 1997;82:3548-52.
9 Ng PC, Wong GWK, Lam CWK, et al. The pituitaryadrenal responses to exogenous human corticotropinreleasing hormone in preterm, very low birth weight infants. F Clin Endocrinol Metab 1997;82:796-9.

10 Ng PC, Blackburn ME, Brownlee KG, Buckler JMH, Dear PRF. Adrenal response in very low birth weight babies after dexamethasone treatment for bronchopulmonary dysplasia. Arch Dis Child 1989;64:1721-6.

$11 \mathrm{Goji} \mathrm{K}$. The corticotropin-releasing hormone test in normal short children: comparison of plasma adrenocorticotropin and cortisol responses to human corticotropin-releasing hormone and insulin-induced hypoglycaemia. Acta Endocrinol 1989;120:390-4

12 Schlaghecke R, Kornely E, Santen RT, Ridderskamps P. The effect of long-term glucocorticoid therapy on pituitary-adrenal responses to exogenous corticotrophinreleasing hormone. N Engl f Med 1992;326:226-30.

13 Wittekind CA, Arnold JD, Leslie GI, Luttrell B, Jones MP Longitudinal study of plasma ACTH and cortisol in very low birth weight infants in the first 8 weeks of life. Early Hum Dev 1993;33:191-200. 